\title{
The ever-expanding breadth of medical expertise
}

Every once in a while it is worth taking a step back to acknowledge how vast the practice of medicine has become. I find myself reflecting on the career of Sir William Osler, sometimes referred to as the "Father of Modern Medicine", and the transformation that has taken place in the medical field since he earned that esteemed title. When Osler graduated medicine in 1872, post-graduate training was informal rather than a requirement to practice. The very notion of medical specialization was still developing in Osler's day. Indeed, in his farewell address to Canadian and American medical students, Osler bemoans the lost "polymathic student", the classic Renaissance man, whose area of study comprised not just the whole of medicine, but the whole of human knowledge.

Fast forward to today, where post-graduate training is not only required, but can be done in any of a dozen specialties and takes up to six years to complete. Subspecialisation through fellowships is now commonplace. As an old saying goes, physicians are now learning more and more about less and less until eventually, they will know everything about nothing.

Yet, this ever-increasing specialisation of knowledge demonstrates how far medical knowledge has advanced from Osler's time. Just as the prototypical Renaissance man became a thing of the past when the world grew too wide for one person to be an expert in all things, so too has medicine grown beyond the limit for any one physician to master all its elements. While a single physician may no longer be a comprehensive source of medical information, physicians and other clinicians working together now have the combined expertise to far surpass what was possible when Osler wrote of the demise of the "polymathic student".

This year's Summer Supplement showcases both the diversity and depth of knowledge which underlies the modern Canadian health care system. Drawing from multiple specialties in medicine, from otolaryngology to oncology, anesthesia to obstetrics, the cases in this issue highlight both how far medical knowledge has come - and how far it still has to go.

- Craig Olmstead

Junior Associate Editor 